\title{
Designing Mathematics Tasks \\ for the Professional Development \\ of Teachers Who Teach Mathematics \\ Students Aged 11-16 Years
}

Debbie Barker and Craig Pournara

\section{Theme}

Our workshop focused on identifying and developing tasks appropriate for use in professional development with mathematics teachers of 11-16 year olds who self-identify as "non-specialists". We invited professional development practitioners, teachers and researchers to join us for a workshop that would involve active engagement with such professional development tasks and a reflection of these tasks from different international perspectives and practice.

\section{Overview}

We explained how our collaboration had started with a meeting in England July 2015 and how we had worked on two "seed activities" which are simple enough to support collaboration but rich enough to be developed for the needs of teachers in our different professional development contexts.

Seed 1-Area of polygons card set: Each card contains a triangle or quadrilateral drawn on a grid. Debbie demonstrated how these could be used as a classroom activity with students. The participants spent time ranking the cards in order of difficulty and discussing the different methods students might use to find the area of each polygon. We then discussed how the task is used during a professional development session. Participants shared their ideas about the activity and their

D. Barker $(\bowtie)$

Mathematics in Education and Industry (MEI), Trowbridge, UK

e-mail: debbie.barker@mei.org.uk 
experiences of using similar activities with students or in professional development. It was agreed that the task is useful as a starting activity for teachers developing their own subject knowledge.

Seed 2-Learning through variation: Following an initial discussion about the ideas underlying variation theory, we worked very briefly to the ideas of variation theory and then worked individually on a series of equations involving square roots, e.g. $\sqrt{x-3}=x-5$. The choices within this example set were made explicit by analysing the dimensions of variation and the range of permissible change. This was followed by examples to explore the number of solutions for equations of the form $\sqrt{x-k}=x-k$, a question posed by teachers in a session with Craig. We acknowledged that while many present could recognise the pattern in the solutions by inspection, it was not likely that non-specialist mathematics teachers would do so. Consequently the task provides opportunity for developing fluency together with the larger purpose of investigating patterns in the solutions.

Finally, Debbie explained how she had used the same seed. At the MEI Conference 2016 she ran a session, The Art of Choosing Examples. This was very much a "beginners' guide" to variation theory and its application in the classroom of the everyday teacher. In contrast to the intended audience of our collaboration, the session was attended by people with a wide range of experience in mathematics education. It was a well-received session and feedback indicated that the ideas of variation are very accessible to teachers and useful in their daily practice. This resonates with Craig's experience in working with teachers in the SA context. There is likely much potential for further supporting teaching and learning using the principles of variation theory.

We were keen for the workshop to be of practical use to delegates, so the session ended with a sharing of a selection of tasks that have been used successfully in professional development with non-specialist mathematics teachers. They were well-received by the participants.

Open Access Except where otherwise noted, this chapter is licensed under a Creative Commons Attribution 4.0 International License. To view a copy of this license, visit http://creativecommons. org/licenses/by/4.0/.

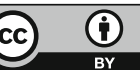

